\title{
Commentary: Blame the sculptors for the heart of stone- Uncovering cellular mechanisms of aortic valve calcification
}

\author{
Daniyil A. Svystonyuk, PhD, and Paul W. M. Fedak, MD, PhD, FRCS(C)
}

\author{
From the Section of Cardiac Surgery Cumming School of Medicine, Department of Cardiac Sciences, University \\ of Calgary, Libin Cardiovascular Institute of Alberta, Calgary, Alberta, Canada. \\ Disclosures: Authors have nothing to disclose with regard to commercial support. \\ Received for publication July 23, 2019; accepted for publication July 25, 2019; available ahead of print Sept 10, \\ 2019. \\ Address for reprints: Paul W.M. Fedak, MD, PhD, FRCS(C), C880, 1403 29th St NW, Calgary, Alberta T2N 2T9, \\ Canada (E-mail: paul.fedak@gmail.com). \\ J Thorac Cardiovasc Surg 2020;159:1754-5 \\ $0022-5223 / \$ 36.00$ \\ Copyright (c) 2019 by The American Association for Thoracic Surgery \\ https://doi.org/10.1016/j.jtcvs.2019.07.074
}

Aortic valve calcification (AVC) is increasingly prevalent in our aging population. The natural history of AVC is characterized by a long asymptomatic latent period, followed by a rapid symptomatic decompensation that is a threat to life. ${ }^{1}$ Aortic valve intervention, either with transcatheter interventions or open surgery, is the only viable therapeutic approach. Disease modifying therapies that prevent or slow AVC may represent an important unmet clinical need to improve outcomes in this growing patient group. ${ }^{2}$

Understanding the mechanistic pathways responsible for AVC and progressive aortic valve degeneration has uncovered a role for valvular interstitial cells (VICs). VICs may be thought of as the "sculptors" of the extracellular environment in both health and disease. Recent preclinical studies have identified that osteogenic differentiation of VICs may underlie progressive AVC; however, the underlying mechanism of how this occurs is deficient. ${ }^{3-5}$ Basic fundamental scientific discoveries may offer new insights into the cellular and molecular basis for AVC. Such foundational knowledge is imperative to the future development of targeted preventive strategies that may halt this seemingly unrelenting process. Predictive tools could also be developed to identify best which individual patients will progress to clinical meaningful aortic valve stenosis and how quickly. The result could be more precise, individualized, and effective treatment strategies.

Accordingly, regulating the osteogenic capacity of VIC may represent an important therapeutic target. In this issue of the Journal, Wang and colleagues ${ }^{6}$ propose a novel molecular mechanism that drives VIC differentiation to an osteogenic fate. Through in vitro studies with human aortic valves, the study shows that human antigen R (HuR) RNA binding protein potentiates MALAT1, a long non-coding RNA that has previously been implicated with promoting tive feedback loop. relevance.

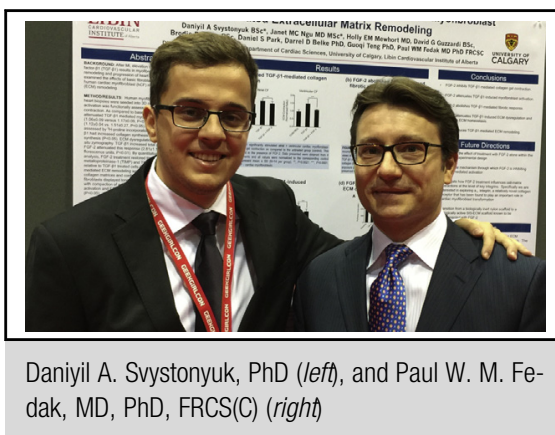

Central Message

Uncovering cellular mechanisms of progressive aortic valve calcification may offer unique insights into potential therapeutic targets and more precise risk prediction.

See Article page 1742.

the osteogenic phenotype. Importantly, Wang and colleagues ${ }^{6}$ uncover that $\mathrm{HuR}$ is downregulated by miR191-3p; however, this microRNA is actively sponged by MALAT1, thereby influencing HuR expression in a posi-

The work of Wang and colleagues ${ }^{6}$ is an important contribution to a growing field of study that explores the osteogenic fate of VICs in the setting of AVC. Their current study is strengthened by the use of primary VICs explanted from human aortic valves. In vitro studies that use single cell populations allow more comprehensive and controlled exploration of the molecular processes that determine cell phenotype. Nevertheless, calcific valvular degeneration is an active process driven by complex interactions among various cellular, biochemical, and hemodynamic stressors. Accordingly, examination of the effects of these stressors on HuR-mediated AVC progression in clinically relevant animal models may be an important next step toward clinical

Uncovering mechanisms of disease is an important step in the translational process. When we encounter a heart that is made of stone, we can be sure that VICs act as the primary sculptors in the aortic valve. The findings by Wang and colleagues ${ }^{6}$ offer the promise that these pathways are highly regulated, and as such, we may be capable of developing tools to prevent these cellular sculptors and limit the calcification process. 


\section{References}

1. Bonow RO, Greenland P. Population-wide trends in aortic stenosis incidence and outcomes. Circulation. 2011;131:969-71.

2. Pawade TA, Newby DE, Dweck MR. Calcification in aortic stenosis: the skeleton key. J Am Coll Cardiol. 2015;66:561-77.

3. Li G, Qiao W, Zhang W, Li F, Shi J, Dong N. The shift of macrophages toward M1 phenotype promotes aortic valvular calcification. J Thorac Cardiovasc Surg. 2017; 153:1318-27.e1.

4. Bouchareb R, Boulanger MC, Tastet L, Mkannez G, Nsaibia MJ, Hadji F, et al Activated platelets promote an osteogenic programme and the progression of calcific aortic valve stenosis. Eur Heart J. 2019;40:1362-73.

5. Bogdanova M, Kostina A, Zihlavnikova Enayati K, Zabirnyk A, Malashicheva A, Stensløkken KO, et al. Inflammation and mechanical stress stimulate osteogenic differentiation of human aortic valve interstitial cells. Front Physiol. 2018;9:1635.

6. Wang Y, Xiao X, Zhou T, Han D, Dong N. Novel mechanisms for osteogenic differentiation of human aortic valve interstitial cells. J Thorac Cardiovasc Surg. 2020;159:1742-53. 